\author{
Marquette University \\ e-Publications@Marquette
}

Biomedical Engineering Faculty Research and

Publications

Biomedical Engineering, Department of

6-2011

\title{
Effect of Ankle Orientation on Heel Loading and Knee Stability for Post-stroke Individuals Wearing Ankle-foot Orthoses
}

M. Barbara Silver-Thorn

Marquette University, barbara.silver-thorn@marquette.edu

Follow this and additional works at: https://epublications.marquette.edu/bioengin_fac

Part of the Biomedical Engineering and Bioengineering Commons

\section{Recommended Citation}

Silver-Thorn, M. Barbara, "Effect of Ankle Orientation on Heel Loading and Knee Stability for Post-stroke Individuals Wearing Ankle-foot Orthoses" (2011). Biomedical Engineering Faculty Research and Publications. 89.

https://epublications.marquette.edu/bioengin_fac/89 
Marquette University

e-Publications@Marquette

\section{Biomedical Engineering Faculty Research and Publications/College of} Engineering

This paper is NOT THE PUBLISHED VERSION; but the author's final, peer-reviewed manuscript. The published version may be accessed by following the link in the citation below.

Prosthetics and Orthotics International, Vol. 35, No. 2 (June 1, 2011): 150-162. DOI. This article is (C) The International Society for Prosthetics and Orthotics and permission has been granted for this version to appear in e-Publications@Marquette. The International Society for Prosthetics and Orthotics does not grant permission for this article to be further copied/distributed or hosted elsewhere without the express permission from The International Society for Prosthetics and Orthotics.

\section{Effect of Ankle Orientation on Heel Loading and Knee Stability for Post-stroke Individuals Wearing Ankle-foot Orthoses}

Barbara Silver-Thorn

Marquette University

Angela Herrmann

Marquette University

Thomas Current

Actra Rehabilitation Associates

John McGuire

Medical College of Wisconsin 


\section{Abstract}

Background: Those who experience lower extremity weakness or paralysis following a stroke often exhibit gait deviations caused by the inability to completely lift their foot during swing. An ankle-foot orthosis (AFO) is commonly prescribed for individuals post stroke with this mobility impairment.

Study design: Randomized controlled trial.

Objectives: To determine whether significant differences could be observed in post-stroke individuals ambulating with an experimental AFO set at three different ankle orientations.

Methods: Gait analysis was conducted for eight post-stroke individuals ambulating with an experimental AFO set in three different randomly selected ankle orientations: $5^{\circ}$ dorsiflexion, $5^{\circ}$ plantarflexion, and neutral alignment. Temporospatial (velocity, cadence, stride length and step length), kinematic (knee angle), kinetic (external knee moment), and plantar force (heel) data were assessed. Within-subject statistical analysis was conducted using the repeated measures ANOVA to determine whether observed differences between the three orientations were significant.

Results: Post-stroke individuals generally exhibited less knee flexion during loading response when their AFO was aligned at $5^{\circ}$ plantarflexion. Six of the eight subjects demonstrated increased knee flexion moment during loading response with the plantarflexed versus dorsiflexed alignment. The plantarflexed ankle orientation also resulted in greater peak heel contact force during loading response.

Conclusions: Post stroke individuals may demonstrate less knee flexion during loading response and increased knee flexion moment (with respect to a dorsiflexed orientation) when their AFO is aligned in $5^{\circ}$ plantarflexion. The fixed plantarflexed ankle orientation consistently resulted in greater peak heel contact force during loading response.

\section{Clinical relevance}

Plantarflexed AFOs are contraindicated for individuals with prior history of pressure sores on their heels. Post stroke individuals placed in $5^{\circ}$ dorsiflexion may demonstrate increased knee flexion, enhanced shock absorption, decreased knee flexion moment, and decreased heel pressure (with respect to a plantarflexed orientation) during loading response.

\section{Keywords}

Ankle foot orthosis, ankle position, knee angle, knee moment, plantar pressure

\section{Introduction}

There are an estimated 5.4 million stroke survivors in the US ${ }^{1}$ who exhibit a variety of mental and physical impairments, including weakness or paralysis on one or both sides of the body and potential gait deviations frequently caused by the inability to completely lift their foot during swing. This deviation, drop foot, results from ankle dorsiflexor weakness and may cause foot slap after initial contact and dragging of the toe during swing. ${ }^{2}$ An AFO designed to assist in maintaining proper foot position and provide mediolateral support to the foot, ankle and subtalar joints is commonly prescribed for individuals with these gait deviations post stroke. ${ }^{3}$

When prescribing and fabricating an orthotic device, the functional goals of the orthosis, degrees of freedom to be altered or affected, forces and bending moments created, and position of the anatomic and orthotic joints must be considered. ${ }^{4}$ For an AFO, ankle position is particularly important. Guidelines regarding AFO ankle position, however, are not well defined.

A study of hemiplegic individuals conducted by Lehmann et al. ${ }^{3}$ indicated that small changes in ankle angle may have significant effects on gait and functional ability. An ankle fixed in excessive dorsiflexion may cause an individual to feel unstable, due to the enhanced flexion moment at the knee and hip during initial contact and 
loading response. ${ }^{3,4}$ In contrast, an ankle fixed in plantarflexion may cause difficulty in limb clearance during swing. ${ }^{3,4}$

In addition to its effects on knee angle, knee flexion moment and knee stability during stance, ankle position may also influence shock absorption and heel loading. ${ }^{4}$ During initial contact, a primary goal of the AFO is to reestablish the heel as the base of support. This facilitates transition to the loading response phase, an eversion response at the subtalar joint, and a knee flexion response during weight acceptance, all of which contribute to shock absorption during early stance. ${ }^{3}$ Positioning the ankle in slight dorsiflexion has been shown to re-establish the heel as the initial contact base of support. ${ }^{3}$

Improving gait (e.g. increased stride length, step length, symmetry and velocity) is an important functional rehabilitation goal for individuals post stroke. ${ }^{5}$ Gait analyses have indicated that step length is increased and stance symmetry (ratio of step lengths of the affected and unaffected sides) is improved in post-stroke subjects when an AFO is used. ${ }^{3,6-13}$ Metabolic energy costs may also decrease significantly when an AFO is used. ${ }^{12}$

The objective of this study was to vary ankle position and observe the overall effect on gait as reflected by temporospatial symmetry, as well as the effects on heel loading and knee stability during loading response, for individuals post stroke wearing a rigid AFO.

\section{Methods}

Eight unilaterally involved post-stroke individuals who were able to actively ambulate within the community were selected for participation in this study (see Table 1). Subject eligibility criteria included at least six months post stroke, unilateral involvement, greater than $10^{\circ}$ passive dorsiflexion with the knee extended (no knee flexion contractures), modified Ashworth scores at the ankle less than 2 (mild to no increased tone), current use of an AFO for ambulation, capability of level walking for 15 metres with an AFO and shoes without use of a cane or walker, and at least 3 months post Botox ${ }^{\circledR}$ injection or never having had a Botox ${ }^{\circledR}$ injection for their lower extremities. Individuals with a history of dizziness, loss of balance, recent falls, cardiac or respiratory disorders that severely limited their activities, and individuals who were pregnant were excluded. Although ankle, knee and hip extensor strength was not assessed, independent ambulation without a cane or walker and no prior incidence of loss of balance or falls indirectly reflected at least modest lower limb muscle strength. The subjects were recruited through physiatrist and orthotist referral. All subjects submitted written informed consent prior to participating in this study, and this protocol was approved by the institutional review boards at both Marquette University and the Medical College of Wisconsin. 
Table 1. Subject Characteristics.

\begin{tabular}{|l|l|l|l|l|l|l|l|l|l|}
\hline & A1 & A2 & ${ }^{*}$ A3 & A4 & A5 & A6 $^{\dagger}$ & A7 & A8 & Mean (sd) \\
\hline Gender & Male & Male & Female & Male & Female & Female & Male & Male & - \\
\hline $\begin{array}{l}\text { Side of } \\
\text { Hemiplegia }\end{array}$ & Right & Left & Right & Left & Left & Left & Left & Left \\
\hline $\begin{array}{l}\text { Current } \\
\text { Orthosis }\end{array}$ & $\begin{array}{l}\text { Pre- } \\
\text { fabricated PA } \\
\text { with DF assist }\end{array}$ & $\begin{array}{l}\text { Custom PNA } \\
\text { (set in } \\
\text { Neutral) }\end{array}$ & $\begin{array}{l}\text { Pre-fabricated } \\
\text { PA with DF } \\
\text { assist }\end{array}$ & $\begin{array}{l}\text { Custom PA } \\
\text { with DF } \\
\text { assist }\end{array}$ & $\begin{array}{l}\text { Custom PA } \\
\text { with DF } \\
\text { assist }\end{array}$ & $\begin{array}{l}\text { Custom PA } \\
\text { with DF } \\
\text { assist }\end{array}$ & $\begin{array}{l}\text { Custom PA } \\
\text { with DF } \\
\text { assist }\end{array}$ & $\begin{array}{l}\text { Custom PA } \\
\text { with DF } \\
\text { assist }\end{array}$ \\
\hline Age (yrs) & 43 & 63 & 60 & 52 & 51 & 49 & 48 & 73 \\
\hline $\begin{array}{l}\text { Time since } \\
\text { stroke (yrs) }\end{array}$ & 3.3 & 1.5 & 24.0 & 1.3 & 3.0 & 8.8 & 3.3 & 5.3 & $54.9(9.8)$ \\
\hline $\begin{array}{l}\text { Body Weight } \\
\text { (kg) }\end{array}$ & 72.6 & 94.8 & 96.1 & 99.8 & 81.0 & 91.2 & 88.5 & 99.8 & $90.5(9.5)$ \\
\hline Height (cm) & 185.4 & 166.4 & 144.8 & 185.4 & 165.1 & 152.4 & 180.3 & 167.6 & $168.4(14.9)$ \\
\hline Selected AFO & $5^{\circ}$ DF & $5^{\circ}$ DF & Neutral & $5^{\circ}$ DF & $5^{\circ}$ DF & Neutral & - & 5 \\
\hline
\end{tabular}

PA - Plastic Articulating, PNA- Plastic Non-articulating, DF - Dorsiflexion.

*Subject A3 had not regularly used her AFO during the past 5 years.

tSubject $A 6$ had calluses on the lateral side of foot. 
An experimental custom-fitted AFO (Figure 1) was fabricated by a certified orthotist (current). Synthetic fibreglass cast material was wrapped around the subject's affected lower leg to create a negative mould. Ankle joints (Becker Motion Control Limiter 655-MCL-L and Becker Tamarack Flexure Joints 742-M-85) were placed on the corresponding plaster positive mould. A thermoplastic sheet of medical-grade polypropylene was draped over the positive mould and vacuum formed. After cooling, the AFO was trimmed and sanded, and Velcro straps and padding then attached.

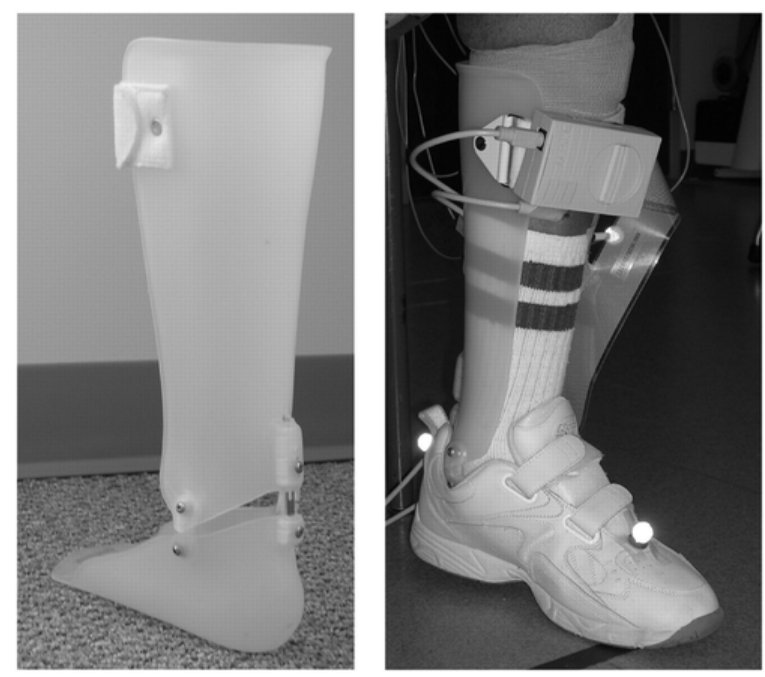

Figure 1. Sample experimental AFO (left) and donned experimental AFO with F-scan sensors/system and passive markers (right).

The orthotist fitted the respective experimental AFO. Each subject was asked to ambulate with the experimental AFO set at each of the three fixed ankle orientations $\left(5^{\circ}\right.$ dorsiflexion, $5^{\circ}$ plantarflexion, or neutral $\left.{ }^{\dagger}\right)$. Slight modifications (e.g. trimming, sanding or flaring) were made to the AFO as needed.

Gait analysis was then conducted at the Human Motion Analysis Laboratory. Kinematic data were collected at $120 \mathrm{~Hz}$ using a video-based Vicon 524 motion analysis system (Vicon Motion Systems, Inc.; Lake Forest, CA) with 15 cameras. The Vicon system was calibrated before each gait analysis session. ${ }^{15}$ Kinetic data from two AMTI ORS6-500 force plates (Advanced Mechanical Technology, Inc.; Watertown, MA) were acquired at $1800 \mathrm{~Hz}$. Plantar force data were collected at $50 \mathrm{~Hz}$ using the F-scan system (Tekscan, Inc.; South Boston, MA), which was calibrated specifically for each subject prior to gait analysis. ${ }^{16}$ Kinetic and kinematic data were synchronized using Vicon hardware.

Anthropomorphic measurements of the subject's height, weight, lower limb segment lengths and girths were collected using an anthropometer. ${ }^{15}$ Reflective markers were placed on the subject's anterior and posterior superior iliac spine, the sacrum, and on the following locations of the affected and unaffected limbs: the lateral epicondyle of the knee, thigh, tibia, and lateral malleolus. ${ }^{15}$ The locations for toe and heel markers were estimated and placed on the subject's shoe as close as possible to the second metatarsal head and calcaneous, respectively. (Knee alignment devices [Motion Lab Systems, Baton Rouge, LA] were placed on the medial and lateral femoral epicondyles of both limbs during a static trial with the AFO in neutral alignment; this triad of markers was used to define the knee joint axis.) Marker locations on the affected leg covered by the AFO were estimated from the unaffected side. To minimize footwear effects, each subject wore the same pair of shoes (heel height less than $1 \mathrm{~cm}$ ) for all walking trials. To acquire plantar force data, thin $(0.15 \mathrm{~mm})$ F-scan sensors were placed between the subject's sock and experimental AFO. 
The subject walked at a comfortable velocity along the corridor $(6.1 \mathrm{~m})$ while kinematic and kinetic data were collected; data were acquired until the subject completed a total of five clean force plate strikes with the affected limb. ${ }^{17}$ This gait analysis procedure was repeated with the experimental AFO set at the remaining ankle orientations.

The raw kinetic and kinematic data for each gait analysis session were first processed in Vicon Workstation (e.g. direct linear transformation of the respective marker locations from each of the 15 individual camera views, marker labelling, and marker motion data filtering using a low pass quintic spline Woltring filter). Gait cycle events (heel strike and toe-off) were labelled for each foot for the gait cycles with clean affected limb contact with the force plate. Velocity, cadence, step length and stride length were computed based on these events. Three-dimensional joint angles and external joint moments (normalized with respect to body mass) were computed using a standard lower-body link segment model (Vicon Bodybuilder) and inverse dynamics calculations. The external knee moment waveforms were used to calculate the peak external knee flexion moment during the loading response phase (affected limb contact through foot-off of the unaffected limb) of the gait cycle for each ankle orientation; Figure 2). The data from each of the five clean dynamic trials were averaged to obtain a single value of peak knee flexion moment and knee flexion angle during loading response, as well as the temporospatial parameters (velocity, cadence, step length and stride length) for each of the three ankle orientations.

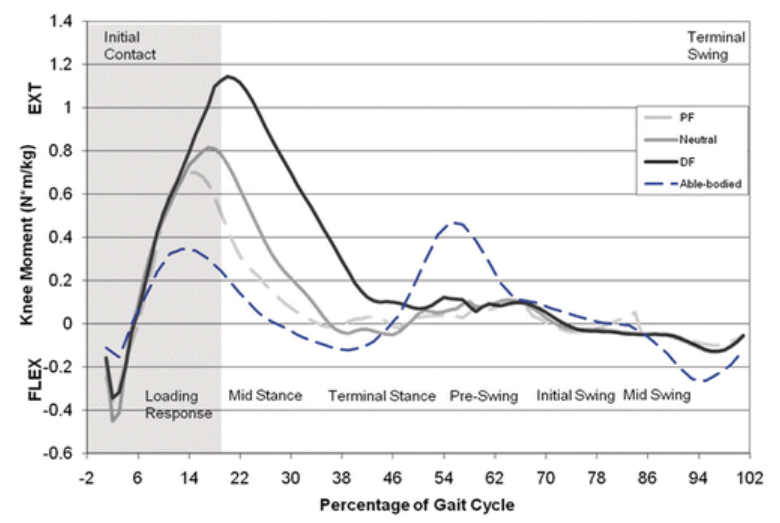

Figure 2. Sample knee moment data (subject A1) at each ankle orientation; able-bodied data ${ }^{15}$ included for reference. PF: $5^{\circ}$ plantarflexed ankle orientation, Neutral: neutral ankle orientation; DF: $5^{\circ}$ dorsiflexed ankle orientation.

F-scan data were acquired during the dynamic trials (a 12-second duration after gait initiation). Five trials of 5-8 gait cycles were used for analysis. The force measured from all sensors within the heel box region were averaged for the middle 2-3 cycles at each ankle orientation to evaluate the mean peak heel force for each of the three ankle orientations for each subject. ${ }^{17}$

An analysis of variance (ANOVA) was conducted across subjects to determine whether observed differences in velocity, cadence, step length and stride length, as well as potential differences in maximum heel force, knee flexion, and knee flexion moment during loading response among the three ankle orientations were significant $(p<0.05){ }^{18}$ Post hoc paired t-tests (Bonferroni correction) were then conducted on variables that demonstrated significant differences to determine which ankle orientations or treatments differed.

The Pearson correlation coefficient ${ }^{18}$ was also computed to assess the dependence of the measured or calculated variables: temporospatial variables, peak knee flexion moment, peak knee angle, and peak heel force during loading response. The correlation (range-1 to +1 ) between respective variables reflects the degree to 
which the variables are dependent, with greater magnitude reflecting a stronger association between the variables.

\section{Results}

All post-stroke subjects ambulated at velocities less than that observed for able-bodied individuals ${ }^{2,19}$ for all ankle orientations, as shown in Figures $3 a$ and $3 b$. While four of the eight subjects $(A 1-2, A 5, A 7)$ demonstrated increased velocity and cadence with the ankle in the neutral orientation, none of these differences was statistically significant (velocity: $p_{\mathrm{ANOVA}}=0.97$; cadence: $p_{\mathrm{ANOVA}}=0.99$ ).
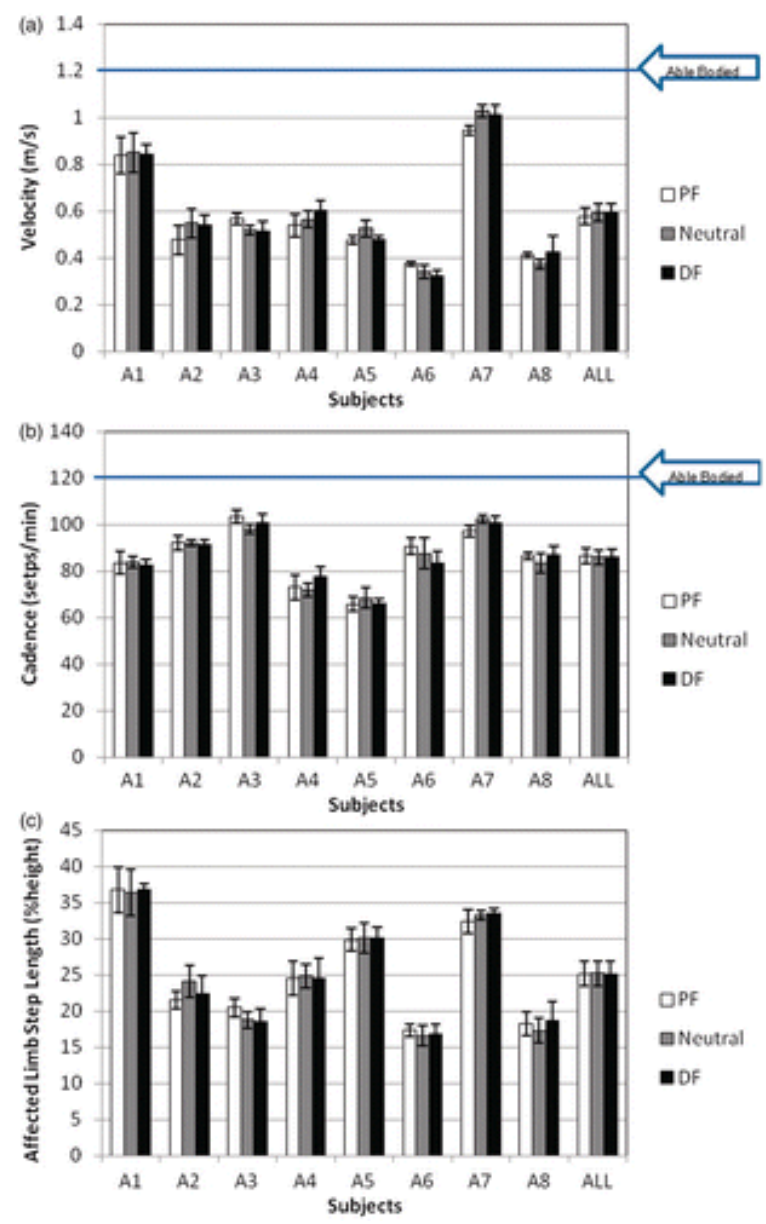

Figure 3. Mean walking velocity (a), cadence (b) and normalized affected limb step length (c) for all subjects (minimum of 5 strides); mean values for the entire subject population also shown; age-matched able-bodied data ${ }^{19}$ for velocity and cadence included for reference. PF: $5^{\circ}$ plantarflexed ankle orientation; Neutral: neutral ankle orientation; DF: $5^{\circ}$ dorsiflexed ankle orientation.

With the exception of subjects A1 and A7, the affected limb step length was less than that observed for ablebodied individuals ${ }^{2,19}$ for all subjects at all ankle orientations (see Figure $3 c$ ). While the step length of the affected limb varied with respect to ankle orientation for all subjects, these differences were again not significant $\left(p_{\mathrm{ANOVA}}=0.99\right)$.

These temporospatial measures can also be used to investigate gait symmetry. A gait cycle may be considered symmetrical if the affected and unaffected steps are of equivalent length (Figure 4). No ankle orientation consistently demonstrated enhanced symmetry for the majority of subjects using this measure. 


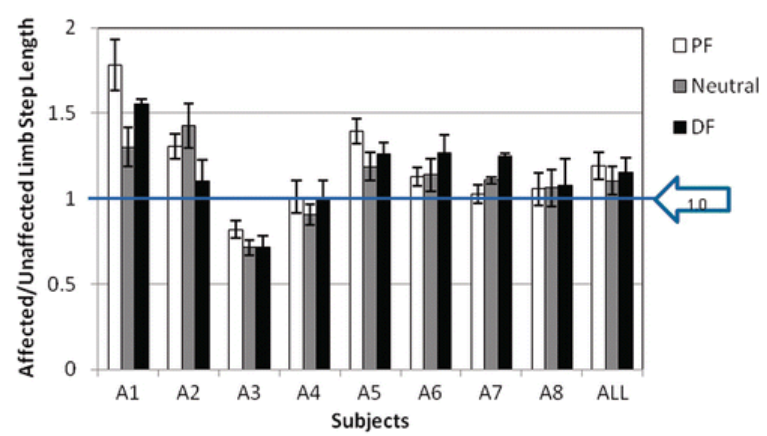

Figure 4. Ratio of affected to unaffected limb step length (minimum of 5 trials, 2-3 gait cycles per trial) for each subject in each ankle orientation; mean value for the entire subject population also shown; a value of unity reflects symmetry. PF: $5^{\circ}$ plantarflexed ankle orientation; Neutral: neutral ankle orientation; DF: $5^{\circ}$ dorsiflexed ankle orientation.

The duration of the loading response phase (e.g. affected limb floor contact until foot-off of the unaffected limb) was also evaluated for each subject. As described by Perry, ${ }^{2}$ the loading response phase for able-bodied individuals typically occurs within the first $10 \%$ of the gait cycle. For the post-stroke population in this study, the loading response period was prolonged, as shown in Figure 5 and observed in prior studies. ${ }^{8,22}$

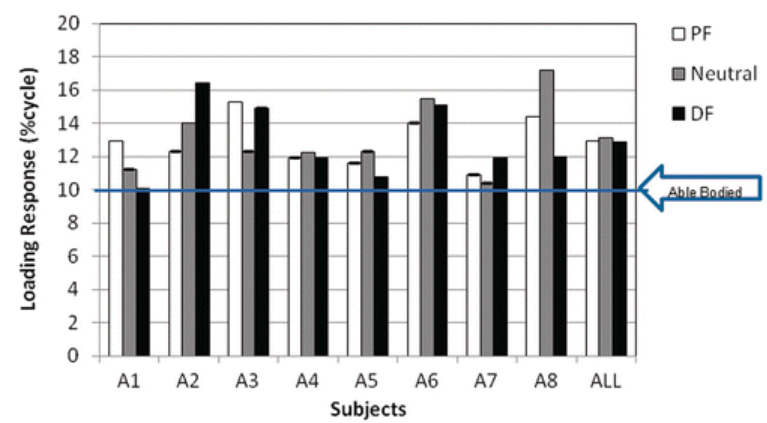

Figure 5. Mean loading response duration (minimum of 5 trials, 2-3 gait cycles per trial) for each subject in each ankle orientation; mean value for the entire subject population also shown; able-bodied data ${ }^{2}$ included for reference. PF: $5^{\circ}$ plantarflexed ankle orientation; Neutral: neutral ankle orientation; DF: $5^{\circ}$ dorsiflexed ankle orientation.

The F-scan system was used to acquire plantar force data for each subject. As the focus of this study was the loading response phase inclusive of initial contact, plantar force analysis was restricted to the heel. The peak heel contact force (Figure 6) was observed within the first $10 \%$ of the gait cycle for all subjects, regardless of loading response duration. Due to pre-existing calluses on the foot of her affected side, subject A6 made incomplete heel contact, as demonstrated by the atypically low heel forces. All subjects demonstrated significantly greater peak heel force in the plantarflexed ankle orientation (post hoc, Bonferroni corrected, paired t-test at the $\alpha=0.05$ level: PF/Neutral $p_{\text {paired } t \text {-test }}=0.012, \mathrm{PF} / \mathrm{DF} p_{\text {paired } \mathrm{t} \text {-test }}=0.055$, Neutral $/ \mathrm{DF} p_{\text {paired } \mathrm{t}}$ test $=0.47)$. Four of the eight subjects $(A 2-3, A 5, A 7)$ demonstrated progressively greater heel force as the AFO orientation moved further into plantarflexion (e.g. dorsiflexion < neutral < plantarflexion). 


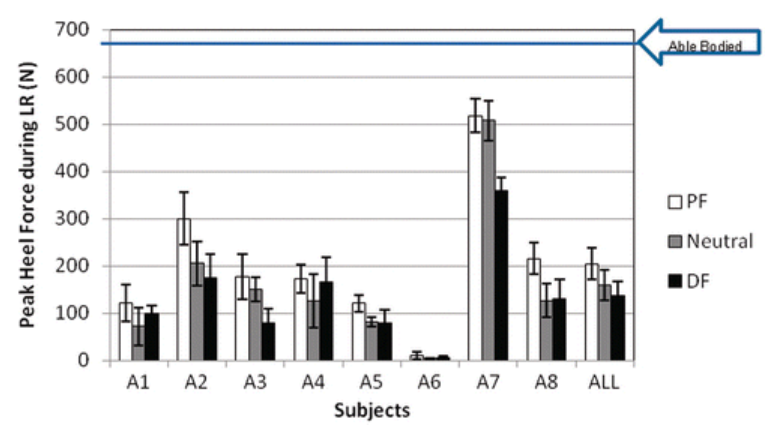

Figure 6. Peak heel force (minimum of 5 trials, 2-3 gait cycles per trial) during loading response for each subject in three ankle orientations; mean value for the entire subject population also shown; able-bodied data ${ }^{16}$ included for reference. PF: $5^{\circ}$ plantarflexed ankle orientation; Neutral: neutral ankle orientation; DF: $5^{\circ}$ dorsiflexed ankle orientation.

In addition to affecting temporospatial measures and heel loading during ambulation, ankle angle also affects proximal limb kinematics. As seen in Figure 7, the maximum knee flexion during the stance phase was observed during loading response, and was less than that of able-bodied subjects ${ }^{2}$ for five of the eight subjects; the remaining three subjects (A2-4) demonstrated increased knee flexion with respect to able-bodied subjects for all ankle orientations. When contrasting the three different ankle orientations within subjects, although all subjects demonstrated decreased knee flexion during loading response in the plantarflexed ankle orientation, these differences were not significant $\left(p_{\text {ANOVA }}=0.67\right.$ ). With the exception of subject $A 5$, knee flexion during loading response was greatest for the dorsiflexed ankle orientation, although these differences were not significant.

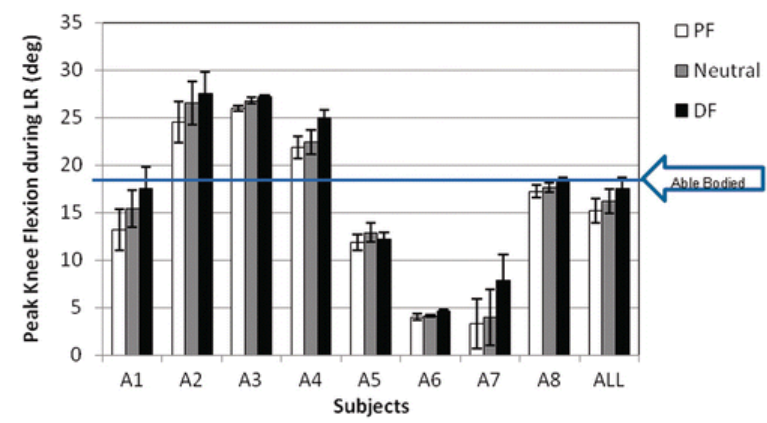

Figure 7. Peak knee flexion during loading response (minimum of 5 trials, 2-3 gait cycles per trial) for all subjects and ankle orientations; mean value for the entire subject population also shown; able-bodied data ${ }^{2}$ included for reference. PF: $5^{\circ}$ plantarflexed ankle orientation; Neutral: neutral ankle orientation; DF: $5^{\circ}$ dorsiflexed ankle orientation.

The maximum initial knee flexion moment, as computed using an inverse dynamics model, was also observed during loading response (Figure 2); these peak external knee flexion moments are shown in Figure 8. Three of the eight subjects (A1, A4, A7) demonstrated greater peak knee flexion moment during loading response compared to able-bodied individuals ${ }^{15}$ in all three ankle orientations, with the neutral orientation exhibiting the greatest knee flexion moment. Three subjects $(A 3, A 5, A 8)$ demonstrated a trend towards reduced peak knee flexion moment (enhanced knee stability) as the ankle orientation progressed to increased dorsiflexion. Six of the eight subjects (all but subjects $A 2$ and A6) demonstrated the smallest knee flexion moment in the dorsiflexed position. While trends were observed, none of these differences was statistically significant $\left(p_{\text {ANOVA }}=0.92\right)$. 


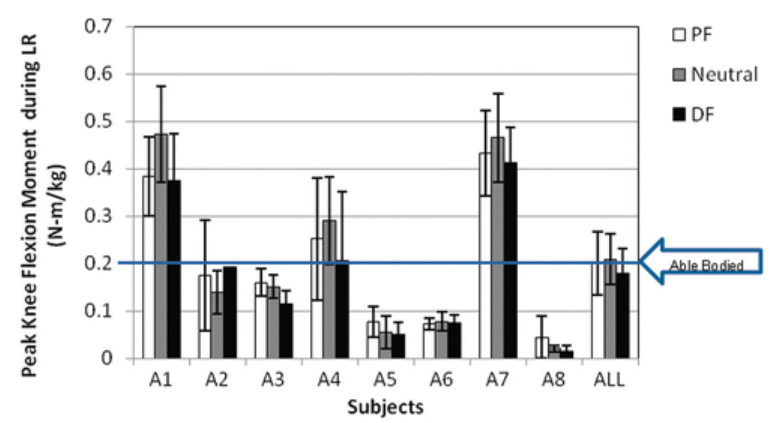

Figure 8. Peak knee flexion moment during loading response (minimum of 5 trials, 2-3 gait cycles per trial) for each subject; mean value for the entire subject population also shown; able-bodied data ${ }^{15}$ included for reference. PF: $5^{\circ}$ plantarflexed ankle orientation; Neutral: neutral ankle orientation; DF: $5^{\circ}$ dorsiflexed ankle orientation.

Each subject was allowed to keep the experimental AFO aligned in their self-selected preferred ankle orientation (see Table 1). Five subjects chose the dorsiflexed ankle orientation, two subjects chose the neutral ankle orientation and one subject declined to keep the experimental AFO.

As shown in Table 2, both velocity and peak knee flexion moment during loading response were significantly correlated with each variable, with the exception of cadence and peak knee flexion angle. The greatest correlation was observed between peak knee flexion moment and velocity. Cadence was found to be significantly correlated with heel force only; peak knee flexion angle was not significantly correlated with any other variable. Heel force had the greatest correlation with velocity and knee flexion moment.

Table 2. Two-tailed Pearson correlation comparison of variables.

\begin{tabular}{|l|r|r|r|r|r|r|}
\hline & \multicolumn{1}{|c|}{ Velocity } & \multicolumn{1}{|c|}{ Cadence } & $\begin{array}{l}\text { Affected limb } \\
\text { Step Length }\end{array}$ & $\begin{array}{l}\text { Knee Flexion } \\
\text { Moment }\end{array}$ & $\begin{array}{l}\text { Knee } \\
\text { Angle }\end{array}$ & $\begin{array}{l}\text { Heel } \\
\text { Force }\end{array}$ \\
\hline Velocity & 1.000 & & & & & \\
\hline Cadence & 0.322 & 1.000 & & & & \\
\hline Affected limb Step Length & $\mathbf{0 . 8 4 6 *}$ & -0.195 & 1.000 & & & \\
\hline Knee Flexion Moment & $\mathbf{0 . 9 3 0 *}$ & 0.281 & $\mathbf{0 . 8 0 2 *}$ & 1.000 & & \\
\hline Knee Angle & -0.220 & 0.047 & -0.244 & -0.173 & 1.000 & \\
\hline Heel Force & $\mathbf{0 . 6 2 0 *}$ & $\mathbf{0 . 4 2 7 * *}$ & 0.364 & $\mathbf{0 . 5 4 8}$ & 0.114 & 1.000 \\
\hline
\end{tabular}

(*Significant at 0.01 level, ${ }^{* *}$ significant at 0.05 level).

\section{Discussion}

Despite the variety of AFO designs available today, there remains a lack of quantitative data concerning prescription criteria. Although an AFO is commonly prescribed to individuals post stroke to aid in lifting the foot during swing, AFOs may cause excessive knee flexion during loading response which may contribute to knee instability. This study used gait analysis techniques to investigate the effect of AFO ankle orientation on temporospatial gait parameters, gait symmetry, heel loading and subsequent knee stability during loading response for individuals post stroke.

Temporospatial gait parameters can be used to assess orthotic performance. ${ }^{20}$ Velocity, cadence, stride length and step length are dependent parameters, as confirmed by the Pearson correlation statistical analysis in Table 2. In this study, these temporospatial parameters were measured as potential functional indicators of overall gait to compare AFO effectiveness at different ankle orientations. Although each parameter was reported separately, their dependence should be acknowledged when assessing gait and inferring gait stability. The temporospatial parameters for each subject were reviewed to assess the ankle orientation that reflected the 
greatest stability during gait. While these temporal parameters varied with ankle orientation, none of the differences were significant based on the ANOVA. Five of the eight subjects opted to keep the AFO that was fixed in $5^{\circ}$ dorsiflexion, perhaps influenced by their prior experience with an articulated AFO with a dorsiflexion assist and/or perceived difficulty in forefoot roll-over (third rocker) in late stance with the neutral and plantarflexed orientations.

Improving gait symmetry is an important functional rehabilitation goal for individuals post stroke. ${ }^{5}$ The effect of AFO ankle orientation on gait symmetry was also investigated in this study, using the ratio of affected to unaffected step length (Figure 4). This measure, however, failed to identify an ankle orientation that consistently resulted in greater gait symmetry; the across-subject differences in this symmetry measure were not significant.

A previous study ${ }^{6}$ investigated gait symmetry for post-stroke individuals ambulating with and without an AFO. Symmetry was improved for post-stroke individuals when an AFO was used, although the specific AFO ankle orientation was not specified. In the current study, the effect of AFO use on gait symmetry was not assessed, as gait analysis was not conducted without an AFO.

Another objective of this study was to contrast the peak heel force, peak knee flexion and knee flexion moment during loading response as a function of AFO ankle orientation for each subject. Loading response addresses three functional objectives: shock absorption, weight-bearing stability and the preservation of forward progression. ${ }^{2}$ For able-bodied individuals, knee flexion during initial contact and loading response is associated with shock absorption. In contrast, for post-stroke individuals who do not use an AFO, the knee typically remains extended during loading response, ${ }^{2,14}$ minimizing potential shock absorption.

For able-bodied individuals, the first part of the foot to make contact with the floor during ambulation is the heel; the heel remains the sole source of support for the first $6-10 \%$ of the gait cycle. ${ }^{2}$ The loading response phase for able-bodied individuals typically occurs during the first $10 \%$ of the gait cycle. ${ }^{2}$ For the post-stroke population examined in this study, the loading response period was prolonged, ranging from 10 to $17 \%$ of the cycle, indicating that toe-off of the contralateral (unaffected) limb is delayed and that affected-limb double support is prolonged. This prolonged loading response is consistent with other studies involving post-stroke individuals. ${ }^{8,22}$

Heel loading as a function of ankle orientation was also investigated. Significant differences in peak heel force during loading response were observed across subjects between the three ankle orientations. Greater peak heel contact forces during loading response were exhibited with the AFO in a plantarflexed position for all subjects. An ankle fixed in $5^{\circ}$ plantarflexion may be expected to result in more rapid loading of the heel during the loading response, potentially reducing shock absorption via knee flexion, as shown previously by Lehmann ${ }^{9}$ and confirmed in Figure 7. A dorsiflexed ankle results in increased knee flexion during stance (as observed for all subjects except A5) and more rapid progression from initial contact to foot flat. This increased knee flexion also makes it difficult to load the heel.

In a related study, Randolph et al. ${ }^{24}$ contrasted plantar pressure at the heel, midfoot and forefoot areas for 10 healthy subjects during ambulation with and without an AFO fixed in slight plantarflexion. Similarly, Crenshaw et al. ${ }^{25}$ investigated plantar pressure of the hindfoot, midfoot and forefoot for 13 healthy subjects during ambulation in a short walking boot aligned in $5^{\circ}$ plantarflexion, neutral, and $5^{\circ}$ dorsiflexion positions. While Randolph observed significant decreases in heel pressure during stance phase during ambulation with the plantarflexed AFO, Crenshaw observed significant increases in hindfoot pressures in the plantarflexed position with respect to both neutral and dorsiflexed alignments. The results of this study were consistent with Crenshaw, whose study design incorporated the same fixed ankle orientations as investigated in this study, and did not contrast an ankle fixed in plantarflexion with a free ankle, as in Randolph's study. 
Lehmann et al. noted that positioning the ankle in slight dorsiflexion re-establishes the heel as the initial contact base of support. ${ }^{3}$ However, this study contrasted peak vertical ground reaction force (GRF) for able-bodied versus hemiparetic subjects only; the effects of AFO ankle orientation and specific heel plantar forces were not investigated. In the current study, four subjects (A1, A4, A6, A8) demonstrated increased heel force during loading response with the dorsiflexed versus neutral ankle orientation, although all subjects demonstrated the greatest heel force during loading response for the plantarflexed ankle orientation.

Three subjects (A2-4) consistently demonstrated greater knee flexion during loading response when compared to able-bodied individuals (Figure 7). Perry ${ }^{2}$ identified four potential causes of excessive knee flexion or inadequate knee extension during loading response: knee flexion contracture, inappropriate hamstring muscle activity, soleus and gastrocnemius weakness, and weak quadriceps. Peat et al. ${ }^{23}$ reported that post-stroke individuals often exhibit greater hamstring activity during loading response when compared to able-bodied individuals. For subjects A2-4, the enhanced knee flexion during loading response may be due to greater hamstring activity or knee flexion contracture. However, electromyogram data were not recorded in this study (the subject selection criteria required greater than $10^{\circ}$ passive dorsiflexion with the knee extended, therefore knee flexion contractures were not a factor).

Five subjects (A1, A5-8) consistently demonstrated less knee flexion during the loading response phase when compared to able-bodied individuals. Perry ${ }^{2}$ identified weak quadriceps as a possible cause of inadequate knee flexion during loading response, as quadriceps activity is necessary to prevent the knee from buckling due to an unstable knee flexion moment. For subjects $A 1$ and $A 5-8$, reduced knee flexion during the loading response when compared to able-bodied individuals may be attributed to weak quadriceps; formal evaluation of hip and knee range of motion and strength is needed to investigate this further.

Finally, although differences in knee flexion during the loading response did not differ significantly, with the exception of subject A5, all subjects demonstrated increased knee flexion during the loading response with the AFO in the dorsiflexed orientation. This result is consistent with Whittle's observation that able-bodied individuals may flex the knee excessively following initial contact if normal plantarflexion of the foot during early loading response is prevented through immobility of the ankle joint. ${ }^{21}$ Additional gait analysis without the use of an AFO may provide further insight.

The peak external knee flexion moment during loading response was used to investigate potential knee stability as a function of AFO ankle orientation. While differences in peak knee flexion moment between the three ankle orientations were observed, ANOVA testing did not demonstrate significance. For an able-bodied gait, the GRF vector is posterior to the knee centre of rotation during initial contact and loading response, contributing to a potentially unstable external knee flexion moment. Stabilization of the knee requires an internal extension moment, usually produced by quadriceps activity. ${ }^{2}$ For able-bodied individuals, a plantarflexed ankle moves the GRF vector anterior to the knee axis of rotation, decreasing the external knee flexion moment during loading response. ${ }^{2}$ In this study, however, only four of the eight post-stroke individuals $(A 1,4,6,7)$ demonstrated decreased knee flexion moment when the affected ankle was fixed in the plantarflexed position (versus the neutral orientation).

Lehmann et al. ${ }^{3}$ also investigated knee flexion moment during gait for hemiparetic individuals ambulating without an AFO and with an AFO fixed in both $5^{\circ}$ dorsiflexion and $5^{\circ}$ plantarflexion (the neutral ankle orientation was not investigated). They observed an increase in the mean total knee flexion moment during mid-stance with the AFO in dorsiflexion versus both plantarflexion and without an AFO. This increase in knee flexion moment with dorsiflexion may appear contrary to the results of this study. However, Lehmann reported total rather than peak knee flexion moment, and these increases were observed during mid-stance (e.g. latter peak in knee flexion moment), rather than during the loading response period (e.g. initial peak in knee flexion moment) as 
investigated in the current study. Only the initial external knee flexion moment during loading response, as reported in this study, has relevance to potential knee instability. Note that the total knee moment reported by Lehmann was based on the relative location of the GRF and the knee axis of rotation, ignoring inertial effects taken into account when using an inverse dynamics approach. His reference to total knee flexion moment refers to the vector sum of knee moment due to the vertical force and the moment due to the fore-aft shear.

A number of potential limitations should be taken into consideration when interpreting the results of this study. Each subject had limited time to adjust to the three ankle orientations, and their prior experience with the different ankle AFO orientations evaluated may have biased the results. For example, as seen in Table 1, subject A3 had not worn her previously prescribed AFO on a regular basis for at least five years; she typically ambulated without an AFO, with her ankle in plantarflexion. The results indicated that, in terms of temporospatial parameters, the $5^{\circ}$ plantarflexed position was most stable for her. Subject A6 also appeared more stable with the ankle in the plantarflexed position; this position provided greater pressure relief from pre-existing calluses on the lateral aspect of her foot. Refining the inclusion criteria to include only subjects who use an AFO to ambulate on a daily basis and excluding individuals with plantar calluses may result in more clinically relevant data free from bias. The current study attempted to minimize the potential influence of AFO alignment familiarity by testing the three ankle orientations in random order. Although intra-subject differences in temporospatial parameters were not significant, further investigation incorporating a definitive acclimation period (perhaps 1-2 weeks) may be warranted.

The current study involved a relatively small subject population. The testing of eight subjects was insufficient to determine significant differences between treatments (ankle orientation) for temporal, spatial, knee moment and heel force data. Subsequent power analyses indicated that a minimum of 24 to 30 post-stroke subjects are needed to detect significant differences in velocity, cadence, stride length, affected limb step length and peak heel force. ${ }^{17}$ As many as 170 subjects may be required to detect differences in peak knee moment during loading response. As such, differences in these parameters may have existed that could not be identified due to the limited sample size.

A final limitation involved the range of ankle orientation ( $5^{\circ}$ dorsiflexion to $5^{\circ}$ plantarflexion) in the experimental AFO used in this study. Expanding the ankle orientation range from $10^{\circ}$ dorsiflexion through $10^{\circ}$ plantarflexion may uncover significant differences in terms of temporospatial gait parameters, although subject selection would likely require modification to ensure subject safety. Future studies should also include gait analysis without an AFO.

\section{Conclusion}

Despite these limitations, the results of this study have important clinical significance. Post-stroke individuals may demonstrate less knee flexion during loading response and increased knee flexion moment (with respect to a dorsiflexed orientation) when their AFO is aligned in $5^{\circ}$ plantarflexion. The fixed plantarflexed ankle orientation consistently resulted in greater peak heel contact force during loading response. AFOs fixed in $5^{\circ}$ plantarflexion are therefore contraindicated for individuals with prior history of pressure sores on their heels. Conversely, post-stroke individuals placed in $5^{\circ}$ dorsiflexion may demonstrate increased knee flexion, enhanced shock absorption and decreased knee flexion moment (with respect to a plantarflexed orientation) during the loading response phase. This dorsiflexed alignment may also relieve force on the heel during the loading response phase.

\section{Funding}

This research received no specific grant from any funding agency in the public, commercial or not-for-profit sectors. 


\section{Conflict of interest}

The authors report no conflicts of interest. The authors alone are responsible for the content and writing of the paper.

\section{Notes}

+Neutral alignment corresponded to an orientation such that the tibia was vertical when the foot was flat on the floor in the AFO and the shoe.

\section{References}

\begin{tabular}{|c|c|}
\hline 1. & American Heart Association. 2005 [online article], http://www.americanheart.org. \\
\hline 2. & Perry, JM . Gait analysis 1992 1st ed. Thorofare, NJ: SLACK Incorporated. \\
\hline 3. & $\begin{array}{l}\text { Lehmann, JF, Condon, SM, Price, R, deLateur, BJ. Gait abnormalities in hemiplegia: their correction by } \\
\text { ankle-foot orthoses. Arch Phys Med Rehabil 1987; 68: 763-771. }\end{array}$ \\
\hline 4. & $\begin{array}{l}\text { Nawoczenski, DA, Epler, ME. Orthotics in functional rehabilitation of the lower } \\
\text { limb } 1997 \text { Pennsylvania: WB Saunder Company. }\end{array}$ \\
\hline 5. & $\begin{array}{l}\text { Hsu, AL, Tang, PF, Jan, MH. Analysis of impairments influencing gait velocity and asymmetry of } \\
\text { hemiplegic patients after mild to moderate stroke. Arch Phys Med Rehabil 2003; } 84: 1185-1193 .\end{array}$ \\
\hline 6. & $\begin{array}{l}\text { Tyson, SF, Thornton, HA. The effect of a hinged ankle foot orthosis on hemiplegic gait: objective } \\
\text { measures and users' opinions. Clin Rehabil } 2001 ; 15: 53-58 .\end{array}$ \\
\hline 7. & $\begin{array}{l}\text { Teasell, RW, McRae, MP, Foley, N, Bhardwaj, A. Physical and functional correlations of ankle-foot } \\
\text { orthosis use in the rehabilitation of stroke patients. Arch Phys Med Rehabil 2001; 82: 1047-1049. }\end{array}$ \\
\hline 8. & $\begin{array}{l}\text { Gok, H, Kucukdeveci, A, Altinkaynak, H, Yavuzer, G, Ergin, S. Effects of ankle foot orthoses on } \\
\text { hemiparetic gait. Clin Rehabil 2003; 17: 137-139. }\end{array}$ \\
\hline 9. & $\begin{array}{l}\text { Lehmann, JF, Condon, SM, de Lateur, BJ, Smith, JC. Ankle-foot orthoses: effect on gait abnormalities in } \\
\text { tibial nerve paralysis. Arch Phys Med Rehabil 1985; 66: 212-218. }\end{array}$ \\
\hline 10. & $\begin{array}{l}\text { Lehmann, JF, Esselman, PC, Ko, MJ, Smith, JC, deLateur, BJ, Dralle, AJ. Plastic ankle-foot orthoses: } \\
\text { evaluation of function. Arch Phys Med Rehabil 1983; 64: 402-407. }\end{array}$ \\
\hline 11. & $\begin{array}{l}\text { Lehmann, JF, Ko, MJ, deLateur, BJ. Knee moments: origin in normal ambulation and their modification } \\
\text { by double-stopped ankle-foot orthoses. Arch Phys Med Rehabil 1982; 63: 345-351. }\end{array}$ \\
\hline 12. & $\begin{array}{l}\text { Danielsson, A, Sunnerhagen, KS. Energy expenditure in stroke subjects walking with a carbon composite } \\
\text { ankle foot orthosis. J Rehabil Med 2004; 36: 165-168. }\end{array}$ \\
\hline 13. & $\begin{array}{l}\text { De Wit, DC, Buurke, JH, Nijlant, JM, ljzerman, MJ, Hermens, HJ. The effect of an ankle-foot orthosis on } \\
\text { walking ability in chronic stroke patients: a randomized controlled trial. Clin Rehabil 2004; 18: 550-557. }\end{array}$ \\
\hline 14. & Rose, J, Gamble, JG(eds) Human walking 1994 2nd ed. Baltimore: Williams \& Wilkins. \\
\hline 15. & Tebbutt, P, Wood, J, King, M. The Vicon Manual 2002 Oxford, UK: Vicon Motion Systems Ltd. \\
\hline 16. & $\begin{array}{l}\text { Murphy, DN, Trachtenberg, G. F-scan educational seminar \& workshop } 2005 \text { Ottawa, Canada . } \\
\text { December } 2005 .\end{array}$ \\
\hline 17. & $\begin{array}{l}\text { Herrmann, A. Effect of ankle orientation on heel loading and knee moment in ankle-foot-orthoses (AFO) } \\
\text { for individuals post stroke. (dissertation) } 2007 \text { Milwaukee (WI): Marquette University. }\end{array}$ \\
\hline 18. & Montgomery, DC . Design and analysis of experiments 2001 5th ed. New York: John Wiley \& Sons Inc. \\
\hline 19. & $\begin{array}{l}\text { Oberg, T, Karsznia, A, Oberg, K. Basic gait parameters: reference data for normal subjects, } 10-79 \text { years } \\
\text { of age. J Rehabil Res Dev 1993; 30: 210-223. }\end{array}$ \\
\hline 20. & $\begin{array}{l}\text { Gard, SA . Use of quantitative gait analysis for the evaluation of prosthetic walking performance. J } \\
\text { Prosthet Orthot 2006; 93: 104-104. }\end{array}$ \\
\hline 21. & Whittle, M. Gait analysis: An introduction 1996 2nd ed. Oxford: Butterworth Heinemann. \\
\hline 22. & $\begin{array}{l}\text { Roth, EJ, Merbitz, C, Mroczek, K, Dugan, SA, Suh, WW. Hemiplegic gait: relationships between walking } \\
\text { speed and other temporal parameters. Am J Phys Med Rehabil 1997; 76: 128-133. }\end{array}$ \\
\hline
\end{tabular}


23. Peat, M, Dubo, HI, Winter, DA, Quanbury, AO, Steinke, T, Grahame, R. Electromyographic temporal analysis of gait: hemiplegic locomotion. Arch Phys Med Rehabil 1976; 57: 421-425.

24. Randolph, A, Nelson, M, deAraujo, M, Perez-Millian, R, Wynn, T. Use of computerized insole sensor system to evaluate the efficacy of a modified ankle-foot orthosis for redistributing heel pressures. Arch Phys Med Rehabil 1999; 80: 801-804.

25. Crenshaw, S, Pollo, F, Brodsky, J. The effect of ankle position on plantar pressure in a short leg walking boot. Foot Ankle Int 2004; 25(2): 69-72. 